\title{
BMJ Open Effects of traditional Chinese exercise on cardiac rehabilitation after percutaneous coronary intervention: study protocol for network meta- analysis of randomised controlled trials
}

\author{
Chuanjin Luo, ${ }^{1}$ Junmao Wen, ${ }^{2}$ Weipeng Sun, ${ }^{2}$ Ting Li, ${ }^{2}$ Xiaojiang Yu, ${ }^{3}$ Tian Zhang, ${ }^{2}$ \\ Xiaoqi Zhou, ${ }^{1}$ Wei Wu, ${ }^{1}$ Rong $\mathrm{Li}^{1}$
}

To cite: Luo C, Wen J, Sun W, et al. Effects of traditional Chinese exercise on cardiac rehabilitation after percutaneous coronary intervention: study protocol for network metaanalysis of randomised controlled trials. BMJ Open 2019;9:e023096. doi:10.1136/ bmjopen-2018-023096

- Prepublication history for this paper is available online. To view these files, please visit the journal online (http://dx.doi. org/10.1136/bmjopen-2018023096).

Received 25 March 2018 Revised 27 November 2018 Accepted 28 November 2018

D) Check for updates

C Author(s) (or their employer(s)) 2019. Re-use permitted under CC BY-NC. No commercial re-use. See rights and permissions. Published by BMJ.

${ }^{1}$ Department of Cardiovascular Disease, First Affiliated Hospital of Guangzhou University of Chinese Medicine, Guangzhou, China

${ }^{2}$ Guangzhou University of Chinese Medicine, Guangzhou, China

${ }^{3}$ Department of Rehabilitation Center, First Affiliated Hospital of Guangzhou University of Chinese Medicine, Guangzhou, China

Correspondence to

Rong Li; 256298797@qq.com

\section{ABSTRACT}

Introduction Coronary heart disease (CHD) is the most common cause of death worldwide. Percutaneous coronary intervention (PCl) has been shown to reduce mortality in patients with $\mathrm{CHD}$. However, there are still recurrences of cardiovascular events after $\mathrm{PCl}$. Cardiac rehabilitation (CR) in patients with established $\mathrm{CHD}$ is associated with reductions in cardiovascular mortality and hospital admissions, as well as improved quality of life. More and more clinical trials suggest that traditional Chinese exercise (TCE) plays a positive role in patients post-PCl. The primary purposes of the current study are to conduct a network meta-analysis of randomised trials to determine the effects of TCE in patients after PCl, and to separately compare the effects of tai chi, baduanjin and yijinjing on CR after PCl.

Methods and analysis Studies will be retrieved from the following databases: PubMed, Embase, Cochrane Library, Chinese National Knowledge Infrastructure, Wanfang Data, Chinese BioMedical Database and Chinese Science and Technology Periodicals Database, from inception to December 2018. We will include randomised controlled trials that are related to the effects of TCE therapies in patients after $\mathrm{PCl}$. The primary outcomes will be all-cause mortality, revascularisations, health-related quality of life and hospitalisations. Two reviewers will independently select eligible articles. For each included article, two reviewers will independently extract the data and assess the risk of bias by using the Cochrane risk of bias tool. Bayesian network meta-analyses will be conducted to pool all treatment effects. The ranking probabilities for the optimal intervention of various treatments (tai chi, baduanjin or yijinjing) will be estimated by the mean ranks and surface under the cumulative ranking curve. The Grading of Recommendations Assessment, Development and Evaluation System will be used to assess the quality of evidence.

Ethics and dissemination The results will be disseminated through peer-reviewed publications. They will provide consolidated evidence to inform clinicians on the potential functions of TCE in CR, and to provide reliable evidence for the application of TCE.

Trial registration number CRD42018088415.
Strengths and limitations of this study

- This is the first systematic review to use network meta-analysis to compare various forms of traditional Chinese exercise (TCE).

- This is the first systematic review to compare the effects of baduanjin, yijinjing and tai chi on cardiac rehabilitation post-percutaneous coronary intervention (PCl).

- This study will assess the effects of TCEs and their safety regarding cardiac rehabilitation after $\mathrm{PCl}$.

- The study will use the Grading of Recommendations Assessment, Development and Evaluation System to further assess the quality of the evidence.

- Due to the changes in the frequency and duration of treatment, there may be methodological heterogeneity.

\section{INTRODUCTION}

Description of the condition

Coronary heart disease (CHD), also known as coronary artery disease, is the most common cause of death worldwide, causing 7.4 million deaths in 2013, accounting for one-third of all deaths globally (WHO 2014). ${ }^{1}$ In addition to the requisite medical therapy, mechanical revascularisation with coronary artery bypass grafting (CABG) or percutaneous coronary intervention (PCI) is needed for patients with CHD. PCI has been shown to reduce mortality in patients with CHD. ${ }^{2}$ During follow-up, however, the benefits of PCI surgery still carry significant risks of coronary spasm, endothelial cell injury, recurrent ischaemia and even restenosis or thrombus. ${ }^{3-5}$ Numerous guidelines endorse the benefits of cardiac rehabilitation (CR), which is recommended for patients with chronic stable angina, acute coronary syndromes or for patients post-PCI surgery. ${ }^{2-10}$ Combined with routine therapy, 


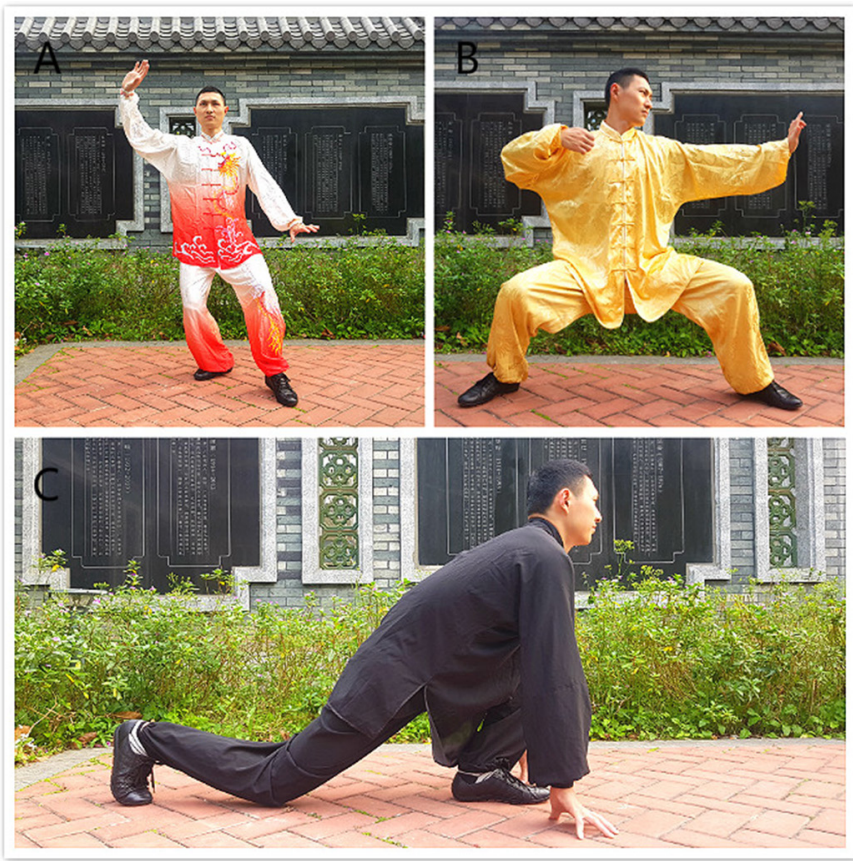

Figure 1 Traditional Chinese exercise ( $A$ is tai chi, $B$ is baduanjin and $\mathrm{C}$ is yijinjing), and the pictured individual has provided consent for publication of their image.

exercise-based CR, which has been recommended by American Heart Association guidelines, ${ }^{11}$ is a safe option. It has been reported that light to moderate intensity physical activity can reduce CHD mortality. ${ }^{12}$

\section{Description of the intervention}

For centuries, traditional Chinese exercise (TCE) (figure 1), a series of mind-body exercises including baduanjin, yijinjing and tai chi (TC, also known as 'taiji chuan'), has been widely practised in China for both preventive and therapeutic purposes. Based on the theoretical principles of traditional Chinese medicine, in clinical rehabilitation focuses on posture, meditation and coordination of breath. The common characteristics of these movements is that they are slow, relaxing and systematic, and therefore suitable for physically

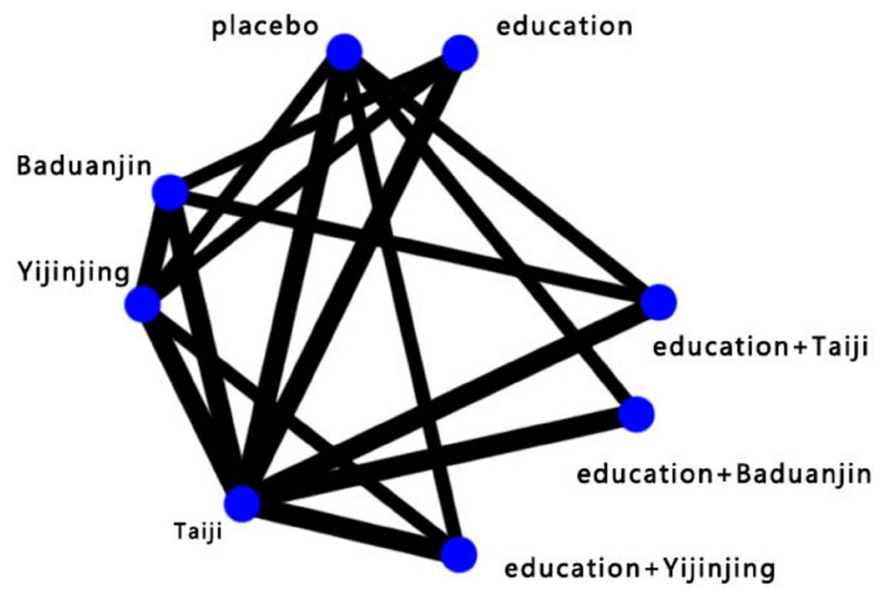

Figure 2 Network plot of all possible direct comparisons between the eligible interventions. weak patients. By correcting body posture and movements, adjusting breathing patterns and maintaining stillness of mind, a wide array of natural self-regulatory/ self-healing mechanisms can be activated to stimulate the balanced release of endogenous neurohormones. ${ }^{13} 14$ Considered to be a low-risk, promising intervention that is used widely in the prevention of cardiovascular disease, TCEs are easy to master in a short time and have few physical demands. ${ }^{15}$ Regardless of previous exercise experience or aerobic capacity, the exercise intensity of TCE is suited for persons of all ages. Moreover, TCE requires no expensive equipment and can be performed either individually or in groups. Most existing systematic reviews have focused on specific forms of TCE, such as TC or baduanjin. ${ }^{16-18}$ Whether TCE has positive clinical effects on patients with CHD after PCI, however, remains unclear.

Therefore, we will conduct the present network meta-analysis (NMA) to explore whether TCE affects the primary endpoint events of cardiac death, recurrence of myocardial infarction (MI), repeated PCI and restenosis after PCI; to determine whether TCE could be an effective method for improving secondary endpoint measures such as quality of life (QoL), physical functions and symptoms, after PCI; to separately compare the effects of TC, baduanjin and yijinjing on patients post-PCI (figure 2).

\section{METHODS AND ANALYSIS Registration}

This protocol has been developed in accordance with the Preferred Reporting Items for Systematic Reviews and Meta-Analyses (PRISMA) statement. It has also been registered with the International Prospective Register of Systematic Reviews. ${ }^{19}$

\section{Eligibility criteria}

\section{Study type}

We will include randomised controlled trials (RCTs) that compare the effects of three different TCE therapies (TC, baduanjin and yijinjing) with a no exercise control on patients with CHD following PCI. The following study designs or publication types will be excluded: (1) non-clinical research literature, such as animal experiments, reviews or case reports; (2) duplicate publications; (3) literature with incomplete data, the study of chaos and (4) studies which lack primary outcome measures. If multiple intervention data can be obtained, the trails can be adopted. If data for comparison of multiple interventions cannot be directly obtained, we will try e-mailing the corresponding author to obtain the original data. If the data cannot be obtained, the trails will also be excluded.

\section{Participants}

This network analysis will include patients with CHD who have received TCE intervention after PCI. 


\section{Interventions}

We plan to include the following TCE therapies: TC, baduanjin and yijinjing, and patients who have received TCE interventions either alone or in combination with appropriate training. For comparisons, both active (eg, walking) or non-active (eg, usual care) controls compared with TCEs will be included. For the reason that some RCTs set a placebo controlled arm as the comparator, information about interventions from placebo controlled trails will also be extracted.

\section{Outcomes}

The primary outcomes will include: (1) all-cause mortality; (2) revascularisations (CABG or percutaneous transluminal coronary angioplasty (PTCA)); (3) results of Medical Outcome Study 36-Item Short-Form Health Survey and (4) hospitalisations: hospital readmission dates.

The secondary outcomes will include: (1) b-type natriuretic peptide; (2) blood lipid indexes (ie, serum total cholesterol, triglyceride, low-density lipoprotein cholesterol and high-density lipoprotein cholesterol); (3) echocardiography and (4) adverse events.

\section{Search strategy}

We will search the following databases: PubMed, Embase, Cochrane Library, Chinese National Knowledge Infrastructure, Wanfang Data, Chinese BioMedical Database and Chinese Science and Technology Periodicals Database. We anticipate that the databases will be searched from their inception to December 2018. The search strategy in PubMed database is as follows: $\ell_{(()((()((T a i)}$ Ji Quan) OR Tai chi) OR Tai ji) OR Taijiquan) OR Baduanjin) OR Baduanjin exercise) OR eight section brocades) OR Yi Jinjing) OR Yijinjing) OR Yijinjing exercise) AND $\left(\left({ }^{(}\left({ }_{(}(()((\right.\right.\right.$Coronary Heart Disease) OR Coronary artery atherosclerosis heart disease) OR CHD) OR Acute coronary syndrome) OR ACS) OR Acute myocardial infarction) OR AMI) OR Heart failure) OR HF) OR percutaneous coronary intervention) OR PCI). The search strategy for PubMed is shown in table 1. The search strategy will be developed by two reviewers (WS and TL) who will independently review the full texts of potentially eligible studies. Discrepancies regarding included studies will be discussed and settled by a third reviewer.

\section{Data collection process}

All titles and abstracts of potential trials will be retrieved and organised by two authors (WS and TL) in Endnote X7 V.7.7.1). Duplicate records will be excluded. The database will then be copied and provided to the first author for duplicate screening. Any disagreements between the two authors will be resolved by discussion with the whole team at a regular meeting. We will allocate the trials to the following five groups: inclusion group, non-patient group, intervention group, outcome group and awaiting group. For this project, Microsoft Excel will be used for preliminary data collection. Four spreadsheets including

\begin{tabular}{cl}
\hline Table 1 & Search strategy used for the PubMed database \\
\hline No. & Search terms \\
\hline 1 & Tai Ji Quan \\
\hline 2 & Tai chi \\
\hline 3 & Tai ji \\
\hline 5 & Taijiquan \\
\hline 6 & Baduanjin \\
\hline 7 & Baduanjin exercise \\
\hline 8 & Yi Jinjing \\
\hline 9 & Yijinjing \\
\hline 10 & Yijinjing exercise \\
\hline 11 & or1-10 \\
\hline 12 & Coronary Heart Diseases \\
\hline 13 & CHD \\
\hline 14 & Coronary artery atherosclerosis heart disease \\
\hline 15 & Acute coronary syndrome \\
\hline 16 & ACS \\
\hline 17 & Acute myocardial infarction \\
\hline 18 & AMl \\
\hline 19 & Heart failure \\
\hline 20 & HF \\
\hline 21 & Cardiovascular events \\
\hline 22 & percutaneous coronary intervention \\
\hline 23 & PCl \\
\hline 24 & or12-23 \\
\hline 25 & 11 and 24 \\
\hline & \\
\hline 14
\end{tabular}

general information (ie, author list, publication year and journal), characteristics of the included trials (ie, diagnostic criteria, age range, study drugs and dose range), risk of bias assessed using the Cochrane risk of bias assessment tool ${ }^{19}$ and outcome data extraction (ie, numbers of response events and non-response events, time points, mean or mean difference, reported outcome definitions and summary data related to treatment effects) will be represented in the table. Relevant original data will be submitted as attachments.

We will express the study selection and data collection process with a PRISMA flow chart (http: //www.prismastatement.org) (figure 3).

\section{Quality assessment}

The methodological quality of the eligible studies, concealment allocation, covering randomisation, blinding and other biases will be evaluated by two authors (TZ and XZ) according to the Cochrane risk of bias assessment tool. ${ }^{20}$ Particular attention will be paid to the adequacy of random allocation concealment and blinding due to the potential failure of inadequate concealment in the randomisation test. Considering the diagnostic criteria, the 


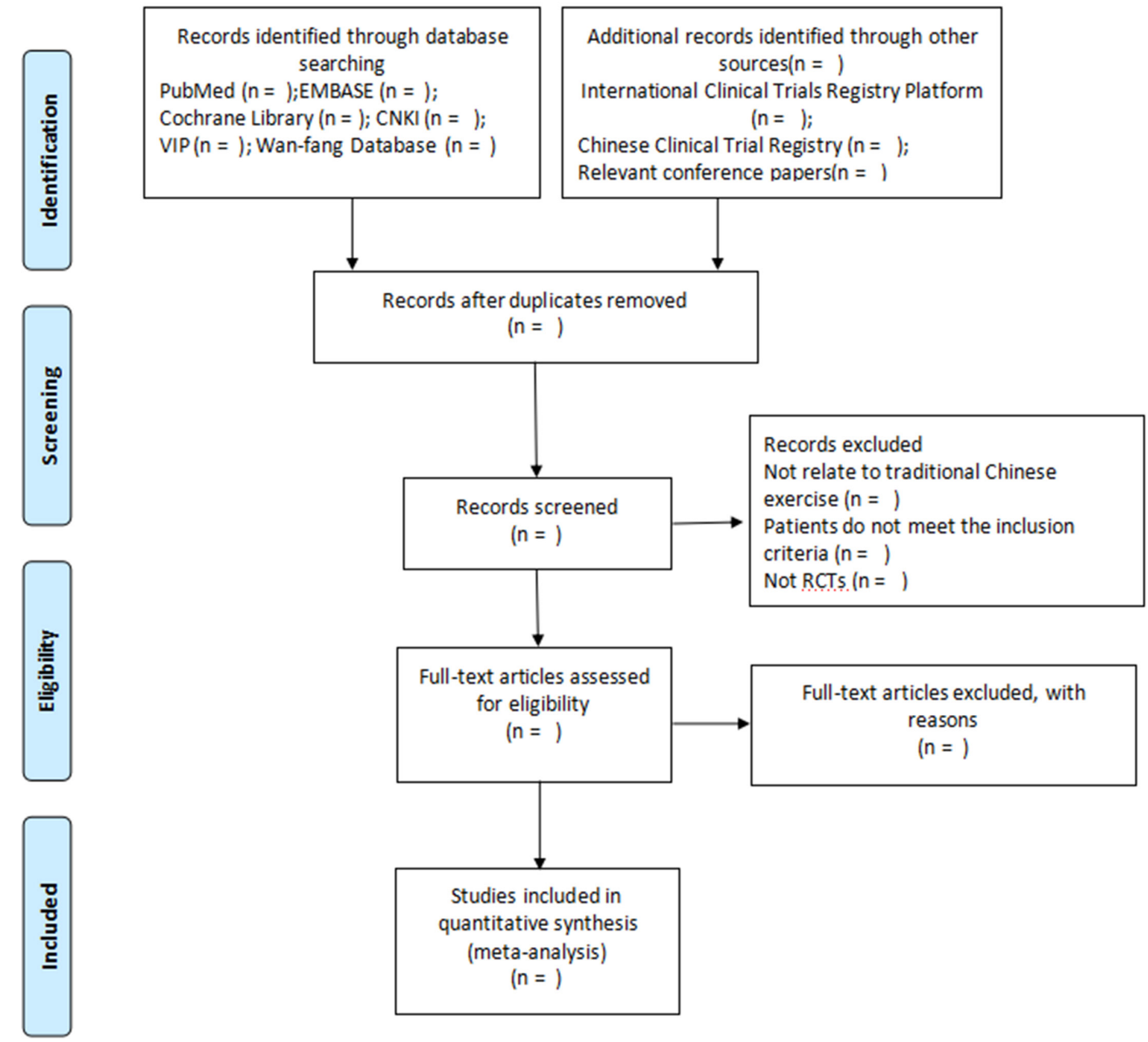

Figure 3 Flow diagram of the study selection process.

sample size calculation method, reporting of withdrawals and follow-up, any other sources of bias will be cautiously assessed. The complete assessment procedures are as follows ${ }^{21}$ : (1) offer direct and indirect effect estimates; (2) assess the quality of direct and indirect estimates; (3) present the results of the NMA and (4) assess the quality of the NMA effect estimates.

\section{Dealing with missing data}

We will initially contact both senior and/or corresponding author to obtain any missing data. If no one responds, the following approaches will be used to estimate the missing data. Instead of providing the mean and SD, the number of responding patients employing a validated imputation method will be calculated for studies failing to report the patients' numbers after treatment. We will also try to estimate from graphs if possible. The reason for exclusion of the available data will be reported.

\section{Network meta-analysis}

We will use the Markov Chain Monte Carlo algorithm by applying WinBUGS 1.4.3 to conduct the NMA in a Bayesian hierarchical framework. The inverse variance heterogeneity method will be used in order to overcome the limitations of the fixed and random effects models. Minimally informative prior distributions will be used for the main outcome. The magnitude of heterogeneity variance will be used to investigate the statistical heterogeneity of all of the NMAs. If direct evidence is feasible, NMAs will be evaluated comprehensively. In treatment effect assessed by indirect and direct comparisons, the potential difference can be evaluated by several methods, and $95 \%$ CIs and z-a values will be calculated for each outcome from each study. The antilogy of the model will be explored by using the node-splitting method, which generates a p-value for the difference between direct and indirect estimates in each closed loop in the network ( $p$-values of $<0.05$ indicate the presence of inconsistency between direct and indirect estimates in a particular closed loop). We will use the deviance information criterion to compare the random and fixed effects models to evaluate model fitness by taking references from the guidelines commonly used in the analogous Akaike Information Criteria: values which decrease by at least 10 points indicate significantly better model fit and parsimony. The mean ranks and surface under the cumulative ranking curve (SUCRA) will be used to sequence the probabilities of the optimal intervention of various treatments. We will describe SUCRA with percentages. For the treatment, better ranks are indicated by higher values. Stata V.12 will be used to generate result figures and NMA graphs. 
If the data cannot be used for quantitative analysis, the evidence will be described and summarised.

\section{Sensitivity analysis and subgroup analysis}

The strategies can be used to address the problem of heterogeneity of pairwise meta-analysis, as well as be employed to tackle incongruity of network analysis. Inconsistent sources will be explored by performing a network meta-regression. We will conduct a sensitivity analysis to explore the strong conclusions of the main results wherever practical. The overall results will be influenced because the methodological quality levels of the studies vary. Sensitivity analysis will be conducted to exclude trials with small sample sizes (ie, arms of less than 10 patients) and remove trails that report the generation of non-random sequences. It is planned (if the number of trials is high enough) to perform sensitivity analysis with respect to age difference and geographical region.

\section{Publication bias}

If feasible, we will also convey any small study effects that exist within a network of interventions. Publication bias will be assessed by performing Egger's regression test. If feasible, a statistical model will be used to convey the network of interventions, including small study effects.

\section{Quality of evidence}

For the main outcomes, two authors (XY and WW) will assess the quality of evidence based on the Grading of Recommendations Assessment, Development and Evaluation, covering study limitations, inconsistency, indirectness, imprecision and publication bias. Five factors, including the limitations of research design, indirectness, incongruity, imprecision and publication bias, will be investigated.

\section{Patient and public involvement}

Patients and public will not be involved.

\section{DISCUSSION}

Previous reviews have indicated that left ventricular (LV) remodelling after MI can be reduced by baduanjin; another study demonstrated that baduanjin provides clinically meaningful improvement for systolic blood pressure (SBP), diastolic blood pressure (DBP), body mass index (BMI), blood glucose, triglyceride (TG), high density lipoprotein cholesterol (HDL-C), low density lipoprotein cholesterol (LDL-C) and QoL. ${ }^{22}$ Research on TC has discovered that it can improve $\mathrm{VO}_{2}$ max in patients with coronary disease. This suggests that TC could be applied in CR as an adjuvant therapy ${ }^{23}$; another study has indicated that TC might improve 6-minute walk distance (6-MWD), QoL and left ventricular ejection fraction (LVEF) in patients, and could be associated with significant reductions in brain natriuretic peptide (BNP) and heart rate (HR). ${ }^{24}$ This would signify the clinical importance of CR post-PCI. Research has also shown that the effects of TCE on blood pressure and blood lipids commonly acknowledged as the primary targets for cardiovascular risk reduction (eg, LDL-C) are clinically significant. $^{25}$

TCE could therefore be used in CR therapy post-PCI, but its efficacy and mechanisms remain unclear. More larger-scale well-designed studies using standardised training protocols are needed in order for specific and accurate conclusions to be made. Thus, the aim of this study is to systematically assess the effects of TCE on patients in CR post-PCI by using enough studies to ensure adequate power for the meta-analysis. To the best of our knowledge, this review will be the first NMA to evaluate the impact of TCE for patients in CR post-PCI. We hope the results of this review will help establish a better approach for exploring the potential functions of TCE in CR and will also provide reliable evidence supporting the use of TCE.

Contributors $C L$ and $R L$ conceived of the study and drafted the protocol. JW, WS and $X Z$ revised it. TL, WW and XY developed the search strategies and conducted data collection. TZ analysed the data independently. All authors have approved the final manuscript.

Funding This study is supported by the 2017 Diagnosis and Treatment Innovation Project at The First Affiliated Hospital of Guangzhou University of Chinese Medicine.

Competing interests None declared.

Patient consent Obtained.

Provenance and peer review Not commissioned; externally peer reviewed.

Open access This is an open access article distributed in accordance with the Creative Commons Attribution Non Commercial (CC BY-NC 4.0) license, which permits others to distribute, remix, adapt, build upon this work non-commercially, and license their derivative works on different terms, provided the original work is properly cited, appropriate credit is given, any changes made indicated, and the use is non-commercial. See: http://creativecommons.org/licenses/by-nc/4.0/.

\section{REFERENCES}

1. Taylor C, Tsakirides C, Moxon J, et al. Submaximal fitness and mortality risk reduction in coronary heart disease: a retrospective cohort study of community-based exercise rehabilitation. BMJ Open 2016;6:e011125.

2. Levine GN, Bates ER, Blankenship JC, et al. 2011 ACCF/AHA SCAI Guideline for Percutaneous Coronary Intervention: executive summary: a report of the American College of Cardiology Foundation/American heart association task force on practice guidelines and the society for cardiovascular angiography and interventions. Circulation 2011;124:2574-609.

3. Zhang Y, Ma XJ, Shi DZ. Effect of trimetazidine in patients undergoing percutaneous coronary intervention: a meta-analysis. PLoS One 2015;10:e0137775.

4. Dzavik V, Ghali WA, Norris C, et al. Long-term survival in 11,661 patients with multivessel coronary artery disease in the era of stenting: a report from the Alberta Provincial Project for Outcome Assessment in Coronary Heart Disease (APPROACH) Investigators. Am Heart J 2001;142:119-26.

5. Kastrati A, Pache J, Dirschinger J, et al. Primary intracoronary stenting in acute myocardial infarction: long-term clinical and angiographic follow-up and risk factor analysis. Am Heart $J$ 2000;139:208-16.

6. Perk J, De Backer G, Gohlke H, et al. European Guidelines on cardiovascular disease prevention in clinical practice (version 2012). The Fifth Joint Task Force of the European Society of Cardiology and Other Societies on Cardiovascular Disease Prevention in Clinical Practice (constituted by representatives of nine societies and by invited experts). Eur Heart J 2012;33:1635-701.

7. Thomas RJ, King M, Lui K, et al. AACVPR/ACC/AHA 2007 performance measures on cardiac rehabilitation for referral to and delivery of cardiac rehabilitation/secondary prevention services endorsed by the American college of chest physicians, american 
college of sports medicine, american physical therapy association, canadian association of cardiac rehabilitation, european association for cardiovascular prevention and rehabilitation, inter-american heart foundation, national association of clinical nurse specialists, preventive cardiovascular nurses association, and the society of thoracic surgeons. J Am Coll Cardiol 2007;50:1400-33.

8. Anderson JL, Adams CD, Antman EM, et al. ACC/AHA 2007 guidelines for the management of patients with unstable angina/non ST-elevation myocardial infarction: a report of the American College of Cardiology/American heart association task force on practice guidelines (Writing committee to revise the 2002 guidelines for the management of patients with unstable angina/Non ST-Elevation myocardial infarction): developed in collaboration with the American college of emergency physicians, the society for cardiovascular angiography and interventions, and the society of thoracic surgeons: endorsed by the American Association of cardiovascular and pulmonary rehabilitation and the society for academic emergency medicine. Circulation 2007;116:e148.

9. Leon AS, Franklin BA, Costa F, et al. Cardiac rehabilitation and secondary prevention of coronary heart disease: an American Heart Association scientific statement from the Council on Clinical Cardiology (Subcommittee on exercise, cardiac rehabilitation, and prevention) and the council on nutrition, physical activity, and metabolism (subcommittee on physical activity), in collaboration with the american association of cardiovascular and pulmonary rehabilitation. Circulation 2005;111:369-76.

10. Eagle KA, Guyton RA, Davidoff R, et al. ACC/AHA 2004 guideline update for coronary artery bypass graft surgery: summary article. A report of the American College of Cardiology/American heart association task force on practice guidelines (committee to update the 1999 guidelines for coronary artery bypass graft surgery). J Am Coll Cardiol 2004;44:e213-310.

11. Fletcher GF, Ades PA, Kligfield P, et al. Exercise standards for testing and training: a scientific statement from the american heart association. Circulation 2013;128:873.

12. Leon AS, Connett J, Jacobs DR, et al. Leisure-time physical activity levels and risk of coronary heart disease and death. The multiple risk factor intervention trial. JAMA 1987;258:2388-95.
13. Wang $X Q$, Pi YL, Chen PJ, et al. Traditional chinese exercise for cardiovascular diseases: systematic review and meta-analysis of randomized controlled trials. J Am Heart Assoc 2016;5:e2562.

14. Jahnke $R$, Larkey L, Rogers $C$, et al. A comprehensive review of health benefits of qigong and tai chi. Am J Health Promot 2010;24:e1-25.

15. Chan CL, Wang CW, Ho RT, et al. A systematic review of the effectiveness of qigong exercise in cardiac rehabilitation. Am J Chin Med 2012;40:255-67.

16. Ding M. Tai Chi for stroke rehabilitation: a focused review. Am J Phys Med Rehabil 2012;91:1091.

17. Dalusung-Angosta $A$. The impact of Tai Chi exercise on coronary heart disease: a systematic review. J Am Acad Nurse Pract 2011;23:376-81.

18. Yeh GY, Wang C, Wayne PM, et al. Tai chi exercise for patients with cardiovascular conditions and risk factors: a systematic review. $J$ Cardiopulm Rehabil Prev 2009;29:152-60.

19. Shamseer L, Moher D, Clarke M, et al. Preferred reporting items for systematic review and meta-analysis protocols (PRISMA-P) 2015: elaboration and explanation. BMJ 2015;350:g7647.

20. Savovic J, Page M, Elbers R, et al. A revised tool for assessing risk of bias in randomized trials (RoB 2.0): TRIALS, 2017:181.

21. Salanti G, Del Giovane C, Chaimani A, et al. Evaluating the quality of evidence from a network meta-analysis. PLoS One 2014;9:e99682.

22. Mao S, Zhang X, Shao B, et al. Baduanjin exercise prevents postmyocardial infarction left ventricular remodeling (be-premier trial): design and rationale of a pragmatic randomized controlled trial. Cardiovasc Drugs Ther 2016;30:315-22.

23. Xiong X, Wang P, Li S, et al. Effect of baduanjin exercise for hypertension: a systematic review and meta-analysis of randomized controlled trials. Maturitas 2015;80:370-8.

24. Yang YL, Wang YH, Wang SR, et al. The Effect of Tai Chi on cardiorespiratory fitness for coronary disease rehabilitation: a systematic review and meta-analysis. Front Physiol 2017;8:1091.

25. Ren X, Li Y, Yang X, et al. The effects of tai chi training in patients with heart failure: a systematic review and meta-analysis. Front Physiol 2017;8:989. 